\title{
Impulsive Noise Cancellation Based on Soft Decision and Recursion
}

\author{
Sina Zahedpour, Soheil Feizi, Arash Amini, \\ Mahmoud Ferdosizadeh, Farokh Marvasti
}

\begin{abstract}
In this paper we propose a new method to recover bandlimited signals corrupted by impulsive noise. The proposed method successively uses adaptive thresholding and soft-decisioning to find the locations and amplitudes of the impulses. In our proposed method, after estimating the positions and amplitudes of the additive impulsive noise, an adaptive algorithm followed by soft-decision is employed to detect and attenuate the impulses. In the next step, by using an iterative method, an approximation of the signal is obtained. This signal approximation is used successively to improve the noise estimate. The algorithm is analyzed and verified by computer simulations. Simulation results confirm the robustness of the proposed algorithm even if the impulsive noise exceeds the theoretical reconstruction capacity.
\end{abstract}

Index Terms - Impulse noise, adaptive systems, error correction, recursive estimation, soft decision.

\section{INTRODUCTION}

$\mathbf{I}$ MPULSIVE noise is a common phenomenon occurring in channels which suffer from switching, manual interruptions, ignition noise, and lightning. Examples of these channels include power line channels [1], Digital Subscriber Line(DSL) systems [2], and digital TV (DVB-T) [3]. Urban and indoor wireless channels as well as underwater acoustic channels also suffer from impulsive noise [4], [5], [6]. In addition to telecommunication channels, sensors used in instrumentations introduce undesired signals such as artifacts and speckles to the measurements [7]. In images, "salt" and "pepper" noise is the most well-known version of the impulsive noise [8]. A Standard Median (SM) filter, is usually preferred for reducing the salt and pepper noise in these 2-D signals [9]. In general, nonlinear methods have shown better performance in reconstruction of signals with impulsive noise [10].

An SM filter manipulates all the samples of the signal, which can cause distortion in other "clean" samples. To address this problem, decision-based algorithms are adopted, in which recovery of corrupted samples is performed after an impulsive noise detection step. One choice to detect the impulses is using hard-decision. As an example, in [11] a hard-decision method based on ordered statistics is used to determine wether a sample is noisy or not. Another choice for detecting impulsive noise is using soft-decision and fuzzy methods [12], [13], [14], [15], [16]. The method in [16], called NASM (Noise Adaptive Soft-switching Method), utilizes both global and local statistics of image pixels. Depending on these

Authors are affiliated with Advanced Communication Research Institute (ACRI), Electrical Engineering Department, Sharif University of Technology \{zahedpour, feizi, arashsil,mferdosi \}@ee.sharif.edu, marvasti@sharif.edu statistics, the estimator switches between Fuzzy Weighted Median (FWM) and SM filters.

Due to impulsive noise, several samples of the signal are lost. The correction of the signal consists of both finding the positions of impulsive noises and estimating the original signal amplitude from the other clean samples. When the location of the impulses (corrupted samples) are known, the problem becomes the same as the erasure channel which is extensively studied in [17], [18] under the subject of interpolation of missing samples. In [19], the more complicated problem of impulsive noise, where error locations are not known, is studied using DFT codes. In fact, the recovery of a bandlimited signal corrupted by impulsive noise is equivalent to decoding error correcting codes when the Galois field is extended to real and complex numbers. To clarify the concept, it should be mentioned that the zero coefficients in the Discrete Fourier Transform (DFT) of real/complex signals play the same role as the parity symbols in the Galois field codes; adding zero coefficients in the DFT domain (sampled frequency domain) is interpreted as oversampling in the time domain [20], [21]. Furthermore, it is also shown that replacement of the DFT with the Discrete Cosine Transform (DCT) would yield similar results [22]. A classical method to locate the position of errors is to compute the Error Locator Polynomial (ELP) and then search for the roots. To show the strength of our method, we compare our results with the ones achieved by this method. In addition to impulsive noise, real/complex signals also contain additive Gaussian noise; thus, sensitivity of the reconstruction methods to this type of noise should also be considered [23]. It is known that iterative methods outperform other techniques when additive Gaussian noise is included [18].

In this paper, we focus on removing additive impulsive noise from a lowpass signal. Since high-pass frequencies (DFT coefficients) of the original signal are zero, respective frequencies of the available signal (original signal combined with noise) are only due to noise. Hence, we can restate our problem as: by having high frequency content of an impulsive noise, we wish to remove it. We employ an iterative method where the initial detection of the impulsive noise locations is performed using an adaptive thresholding technique similar to the one used in a radar's Constant False Alarm Rate (CFAR) [24] detector. We refer to the process of finding the impulsive locations as detection. The result of CFAR thresholding is used to make a soft-decision on whether the sample is corrupted or not. Subsequently, these information are used in the interpolation of the signal amplitudes using an iterative method (referred as signal estimation). The latter estimate is in turn used to 


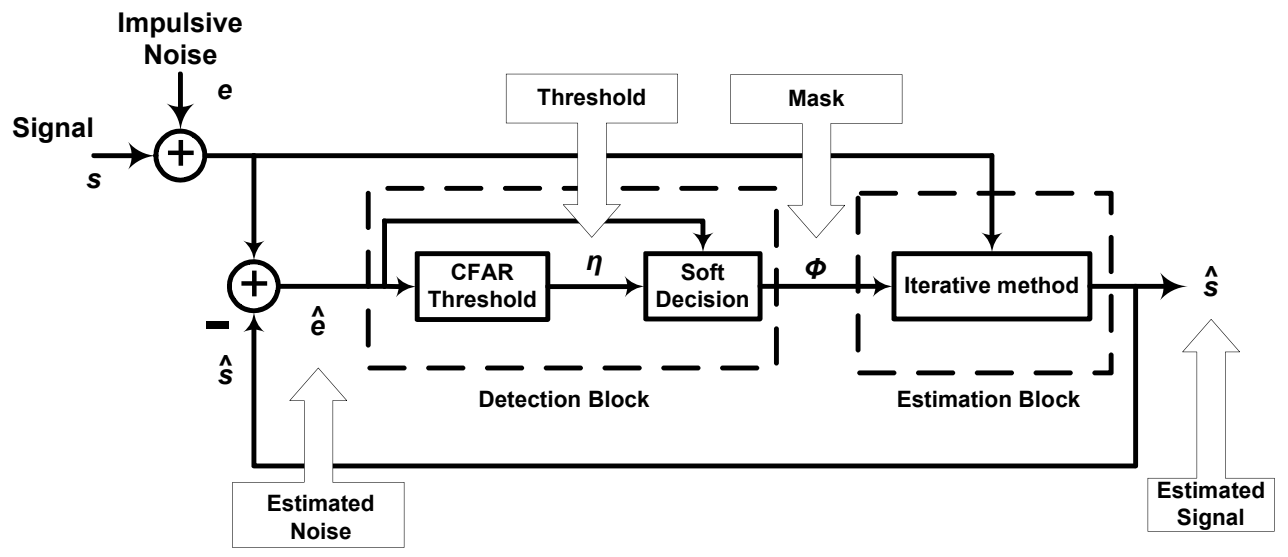

Fig. 1. The Recursive Detection-Estimation (RDE) method.

improve the detection process while the output of the improved detection is again fed to the estimation block. As this detection and estimation loop continues, we get better approximates of the signal.

The rest of this paper is organized as follows: In section II the proposed method is introduced. The detection and estimation blocks are discussed in sections III and IV, respectively, while in section $\mathrm{V}$ the system is analyzed mathematically. In sections VI and VII alternative methods are presented and compared. Computer simulation results are discussed in section VIII. Finally, section IX concludes the paper.

\section{The Proposed Method}

In this paper, we focus on removing additive impulsive noise $(e[i])$ using the zeros in the DFT domain. To be specific, we define impulsive noise as an $n$-point discrete signal where except for $m$ samples $(m<n)$, the rest are zero. Any Probability Distribution Function (PDF) can be assumed for the values of the $m$ nonzero samples; however, we assume Gaussian distribution for ease of analysis. Furthermore, the locations of these $m$ nonzero samples are taken to be uniformly distributed among all the $n$ samples. In some applications, instead of uniform-Gaussian distribution, other models such as the Bernoulli-Weibull [25] and Middleton's Class-A noise model [26] are employed.

We assume that the original signal $(s[i])$ is real, which implies that its DFT is conjugate symmetric. Since for the reconstruction of each impulsive noise we need two pieces of information (location and amplitude), we require at least two independent equations which could be obtained by one zero in the DFT domain; each zero describes two linear equations between time domain samples, one regarding the real part and one for the imaginary part. However, due to the mentioned symmetry, each zero DFT coefficient in the positive frequencies is coupled with a zero in the negative frequencies which do not yield new equations. Thus, the "Error Correction Capacity" of a given block (maximum number of corrupted samples we should be able to reconstruct) can be defined as half the number of its zeros in the DFT domain; i.e., if the signal contains $n_{z}$ zeros in the DFT domain the error correction capacity is $n_{z} / 2$. In this paper error correction capacity and reconstruction capacity are used interchangeably. The terms full capacity and half capacity indicate the scenarios where the number of samples corrupted by impulsive noise is equal to or half the reconstruction capacity, respectively.

Figure 1 depicts the block diagram of our proposed method. As this figure suggests, the detection of impulse locations and estimation of signal amplitudes are used successively in a loop; this method is called Recursive Detection-Estimation (RDE). To distinguish between the two different iterative methods used in this paper, we use the term "RDE step" for each time the detection-estimation loop proceeds (which should not be confused with iterations of the iterative method shown in Fig. 1). In each RDE step, the noise estimate $(\hat{e})$ is improved using the approximated signal $(\hat{s})$ resulted from the previous RDE step by subtracting the estimated signal $(\hat{s})$ from the noisy one:

$$
\hat{e}_{\text {new }}[i]=r[i]-\hat{s}[i]
$$

The detection block functions as follows: If the amplitudes of impulsive noise are large enough, corrupted samples could be distinguished from the neighboring samples; thus, each detection is based on the difference of the sample and the average of its neighbors. This method is similar to the CFAR algorithm used in radar detectors. Hence, the detection block yields an adaptive threshold $(\eta)$ using the impulsive noise estimate. As an example, the following equation describes the threshold obtained by the simple CFAR algorithm (not used in our method):

$$
\eta_{\text {simple }}[i]=\frac{1}{2 n_{c}}\left(\sum_{j=1}^{n_{c}}\left|\hat{e}_{\text {new }}[i-j]\right|+\sum_{j=1}^{n_{c}}\left|\hat{e}_{\text {new }}[i+j]\right|\right)
$$

Based on the resultant threshold, $\eta$, we can decide upon noisiness of a sample either by hard-decision or by softdecision. In the hard-decision, a sample is regarded as noisy when its amplitude is greater than the threshold and vice versa. Alternatively, in our soft decision scheme, instead of two possible labels "noisy" and "clean", we attenuate each 
sample with respect to its distance from the threshold $(|\hat{e}-\eta|)$ which is in fact a measure of our certainty about its noisiness. As the difference between a sample and the adaptive threshold increases, the attenuating factor decreases to zero, which implies that the sample is likely to be noisy; we denote the vector of the attenuating factors by mask $(\phi)$ and the attenuation factor of each sample is called the respective mask coefficient $(\phi[i])$ :

$$
\text { masked noisy signal }=\phi[i] \times r[i], \quad 1 \leq i \leq n
$$

In each RDE step, as the accuracy of the impulsive noise estimate improves, we obtain a new mask. Computer simulations confirm that in initial RDE steps, the soft-decision method leads to better outputs than the hard-decision method. As the number of RDE steps increase, better estimates of the impulsive noise is obtained. This makes it possible to shift the soft-decision threshold to the hard one (using a parameter of $\phi$ called $\alpha$ ), which in turn makes the mask more accurate.

After detecting the impulsive noise locations, the amplitude of the corrupted sample is estimated. The estimation is based on interpolation of the amplitude using the surrounding clean samples. Multiplying the noisy signal by the mask and filtering the result is a simple method to approximate the original signal. Although this simple method is likely to alleviate the noise effect, it certainly distorts other clean samples. To compensate for the distortion, we use an iterative method (shown as "iterative method" in Fig. 1) inside the estimator in each RDE step. To clarify the roles of RDE loop and the iterative method, it should be emphasized that the RDE steps improve the detection of corrupted samples (locations) while the iterative method enhances the estimation of original signal amplitudes. The algorithm of the proposed method can be summarized as follows:

1) Approximate the impulsive noise (ê) by subtracting the estimated signal from the noisy one (1); in the first RDE step, since no estimate about the signal is available, signal is assumed to be zero.

2) Generate thresholds, $\eta$, using CML-CFAR discussed in section III-A.

3) Generate a mask, $\phi$, using the soft-decision detector (with parameter $\alpha$ ) discussed in section III-B.

4) Use the mask in conjunction with the iterative method introduced in section IV to improve the estimate of the signal.

5) Return to the first step. In the soft-decision function of step 3, increase parameter $\alpha$ to shift the soft-decision function to the hard one. Increase the number of iterations in step 4 to further improve the signal estimation.

In the next two sections, details of the detection and estimation blocks are discussed.

\section{IMPUlsive NoISE Detection}

As previously described, we introduce a multiplicative mask in order to suppress the impulses, while trying to keep the clean samples unchanged. Since we only have an estimate of the impulse locations, two types of errors are likely to happen. A missed detection occurs if a corrupted sample is not

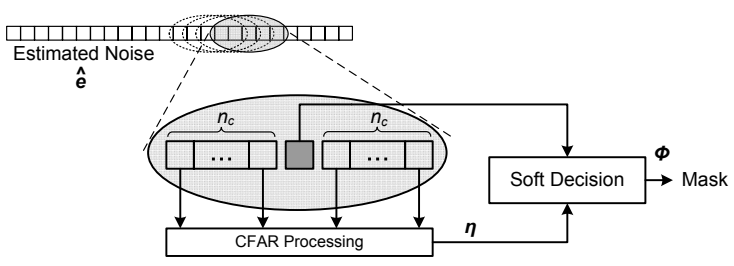

Fig. 2. A typical CFAR detector and decision block.

detected, while a false alarm occurs if a legitimate sample is detected as noise. Two important techniques are incorporated to alleviate these effects: 1- The use of CFAR and 2- the employment of soft-decision. We explain these techniques in the following subsections.

\section{A. Adaptive Impulsive Noise Detection Using CFAR}

The threshold which is used to detect impulsive noise can either be adaptive or non-adaptive. In the non-adaptive method, a fixed threshold is used to compare the amplitude of the sample under scrutiny to determine whether an impulse at that instant exists or not. This threshold is obtained using the statistical properties of the signal and noise. On the other hand, the more sophisticated thresholding schemes incorporate the amplitude of the adjacent samples in order to obtain adaptive thresholds. The CFAR algorithm is an example of an adaptive thresholding method used in radar detectors based on Neyman-Pearson criterion [27]. In radar applications, the detector detects the target in the presence of noise and clutter, which is equivalent to the detection of impulsive noise locations among other clean samples. Thus the techniques used in radar detectors can be adopted for the problem at hand. Figure 2 depicts a simple CFAR detector. The CFAR processing block may be either amplitude averaging (Cell Averaging-CFAR)as suggested in (2) or a more complicated combination of adjacent samples. In this paper we use a Censored Mean Level (CML) CFAR. In the $k^{t h}$ order CML-CFAR of length $2 n_{c}$, among the $2 n_{c}$ adjacent cells, $k$ of the smallest amplitudes are averaged and the other $2\left(n_{c}-k\right)$ samples (which may contain impulses) are ignored. In other words, if $\left|\hat{e}\left[j_{1}\right]\right| \leq \cdots \leq\left|\hat{e}\left[j_{2 n_{c}}\right]\right|$ where $\left\{j_{1}, \cdots, j_{2 n_{c}}\right\}=\left\{i-n_{c}, \cdots, i+n_{c}\right\} /\{i\}$, we have:

$$
\eta[i]=\frac{1}{k} \sum_{l=1}^{k}\left|\hat{e}\left[j_{l}\right]\right|
$$

The reason we use CML-CFAR is to reduce the probability that impulses contribute in the averaging when some of them are present in adjacent cells.

\section{B. Soft-decision Vs. Hard-decision}

The detection stage is not an error-free process in the early RDE steps, especially when the amplitudes of the impulses are small compared to the signal amplitudes. When the harddecision method is employed, the mask is either zero or one at each sample; thus some samples are erased. The optimistic point of view is that only the noisy samples are lost; however, if we have mistakenly discarded a number of clean samples 
(resulting in false alarms), this error may or may not be recoverable depending on the number of impulses with respect to the number of zeros in the DFT domain. On the other hand, when we use soft-decision, even when the detector makes a number of false alarms, the attenuation can be compensated without any need to extra zeros in the DFT domain. The disadvantage of the "soft" method is the low convergence rate in the signal estimation block even when good estimates of the impulsive noise locations are available. To overcome this drawback, we gradually change the soft-decision to the hard threshold as the number of RDE steps (estimation fidelity of the noise locations) increases. To summarize, using hard-decision within the detection block, a binary mask is generated; i.e, the value of the mask at each sample is zero if the sample is detected as noisy and one otherwise. On the other hand, the soft-decision block generates a real number between zero and one depending on the certainty of the detector.

Simulation results suggest that the mask function of the form

$$
\phi(\hat{e}[i], \eta[i])=e^{-\alpha|\hat{e}[i]-\eta[i]|}
$$

performs well, where $\hat{e}$ is the estimated error, $\eta$ is the threshold generated by CFAR [28] and $\alpha$ is the softness order of the detection block. The suggested $\phi(\hat{e}, \eta)$ approaches one as $\hat{e}-\eta$ tends to zero. The parameter $\alpha$ is increased gradually through RDE steps in order to change the soft-decision to the harddecision which in turn increases the convergence rate of the estimator.

\section{Signal ESTIMATION}

In the rest of this paper, we assume the original signal, $s[i]$, is discrete and lowpass with $n_{z}$ zeros in the DFT domain. Thus, ideally we should be able to detect and reconstruct $n_{z} / 2$ corrupted samples. Mathematically, we should solve an underdetermined system of equations [29] with some additional constraints (number of impulses do not exceed $n_{z} / 2$ and each impulse corrupts only one sample). Let $r[i]=s[i]+e[i]$, where $r[i]$ is the noisy signal, $s[i]$ is the original signal and $e[i]$ is the impulsive noise. We have

$$
\left[\begin{array}{l}
R 1 \\
R_{2}
\end{array}\right]=\left[\begin{array}{c}
S_{1} \\
0
\end{array}\right]+\left[\begin{array}{c}
E_{1} \\
E_{2}
\end{array}\right]=\left[\begin{array}{c}
S_{1}+E_{1} \\
E_{2}
\end{array}\right]
$$

where the capital letters denote the respective signals in the DFT domain. $R_{1}, S_{1}$ and $E_{1}$ are the lowpass components of the $R, S$ and $E$ signals, respectively, while $R_{2}$ and $E_{2}$ are the highpass parts (the highpass component of $S$ is zero.). $E$ and $e$ are related to each other by the DFT matrix $(\Delta)$ as

$$
E=\Delta_{n \times n} \cdot e
$$

where

$$
\boldsymbol{\Delta}=\left(\begin{array}{ccccc}
1 & 1 & 1 & \ldots & 1 \\
1 & e^{-j \frac{2 \pi}{n}} & e^{-j \frac{2 \pi}{n} 2} & \ldots & e^{-j \frac{2 \pi}{n}(n-1)} \\
\vdots & \vdots & \vdots & \ddots & \vdots \\
1 & e^{-j \frac{2 \pi}{n}(n-1)} & e^{-j \frac{2 \pi}{n} 2(n-1)} & \ldots & e^{-j \frac{2 \pi}{n}(n-1)^{2}}
\end{array}\right)
$$

To reconstruct the signal, we should solve the underdetermined system of equations

$$
E_{2}=\Delta_{n_{z} \times n} \cdot e
$$

where $\boldsymbol{\Delta}_{n_{z} \times n}$ contains the $n_{z}$ rows of $\boldsymbol{\Delta}$ which are zero in $S$. In subsection IV-A we introduce a general iterative method to synthesize the inverse of a system. As mentioned in section II, these iterations are performed in the estimation block to approximate the original signal (in each RDE step) and should not be confused with RDE (external) loop.

\section{A. Successive Signal Approximations Using an Iterative Method}

The iterative method introduced in [18] is a general approach to approximate the inverse of a class of invertible operators in a finite number of iterations. Furthermore, for a wider class of invertible and linear non-invertible operators, convergence of this method to the pseudo-inverse solution has been shown [30]. The invertible operator can be non-linear and/or time varying.

We denote the operator that we intend to find its inverse by $G$. Also, $y=G \cdot x$ means that $y$ is the output of the operator when $x$ is the input. The purpose of the iterative method is to estimate $x$ when $y$ and $G$ are known; i.e., approximating $G^{-1}$. Using the operator algebra and denoting the identity operator by $I$, we have:

$$
G^{-1}=\frac{\lambda}{I-(I-\lambda G)}=\lambda \sum_{i=0}^{\infty}(I-\lambda G)^{i}
$$

The assumption of $\|I-\lambda G\|<1$ is necessary for convergence, where $\|\cdot\|$ is the $\ell_{2}$ norm. Thus,

$$
x=\lambda \sum_{i=0}^{\infty}(I-\lambda G)^{i} \cdot y
$$

The output of each iteration step is equivalent to truncating the above series. The output of the $(k+1)^{t h}$ iteration is

$$
\begin{aligned}
x^{(k+1)} & =\lambda \sum_{i=0}^{k+1}(I-\lambda G)^{i} \cdot y \\
& =\lambda y+(I-\lambda G) \cdot \lambda \sum_{i=0}^{k}(I-\lambda G)^{i} \cdot y
\end{aligned}
$$

Since $y=G \cdot x,(12)$ can be written recursively:

$$
x^{(k+1)}=x^{(k)}+\lambda G \cdot\left(x-x^{(k)}\right)
$$

In the following, we refer to (13) as the iterative method. It is obvious that the condition $\|I-\lambda G\|<1$ is met only for a specific range of $\lambda$ (this range is normally $0 \leq \lambda<2$ ); moreover, decreasing $\|I-\lambda G\|$ by increasing $\lambda$ will result in a faster convergence rate. Hence, $\lambda$ determines whether the method converges and if it does, how fast it converges. The block diagram of (13) is shown in Fig.3 (a). For our special case in this paper, we briefly present the proof of the convergence for a linear contraction operator $G(\|G\|<1)$. In order to show this, assume

$$
z^{(k)}=x-x^{(k)}
$$




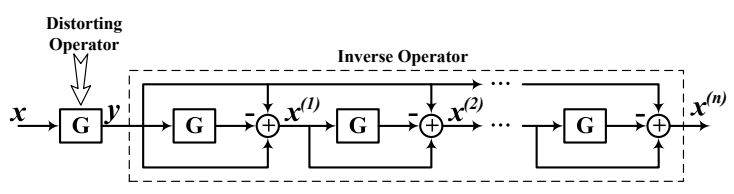

(a)

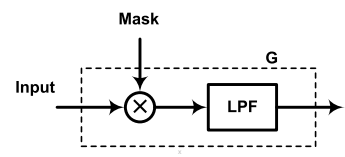

(b)

Fig. 3. (a) Block diagram of the iterative method with $\lambda=1$ (b) Model of the distortion block $(\mathrm{G})$ in our specific application.

thus

$$
z^{(k+1)}=x-x^{(k+1)}=z^{(k)}-\lambda\left(G \cdot\left(x-x^{(k)}\right)\right)
$$

or

$$
z^{(k+1)}=D \cdot z^{(k)}
$$

where $D=I-\lambda G$. For $0<\lambda<2$, using the contraction property of $G$ we have $\|D\|<1$ and therefore $z^{(k+1)} \rightarrow 0$, which results in $x^{(k)} \rightarrow x$.

Below, we apply the iterative method introduced above to estimate the amplitude of the corrupted samples using the mask generated by the detection block as part of the $G$ operator. The detection block introduced in section III, generates a mask vector which contains information about the locations of the impulsive noise. By employing the mask, the iterative method described by (13) can be used to recover the corrupted samples. The distortion operator applied for these iterations is depicted in Fig. 3 (b). In addition to the mask, a lowpass filter is employed which helps the reconstruction of the corrupted samples. Both the mask and the lowpass filter are linear contraction operators; consequently, the depicted distortion block is also a linear contraction which validates the convergence to the solution (section IV-A). In the early RDE steps, since the outputs of the detection block are not reliable, a small number of iterations are used. In contrast, when better approximations of the signal are obtained (at higher RDE steps), the increase in the number of iterations yields in better performance.

In the next section, a mathematical analysis of the proposed method is discussed.

\section{Analysis of the Proposed Method}

In this section, the effect of the iterative method on both the signal and the impulsive noise is investigated. Since $G$ in our application is linear, its operation can be modeled in general by multiplication of a matrix $\mathbf{G}$ :

$$
\mathbf{G}=\boldsymbol{\Delta}^{-1} \cdot \operatorname{diag}(\underbrace{1, \ldots, 1}_{n-n_{z}}, \underbrace{0, \ldots, 0}_{n_{z}}) \cdot \boldsymbol{\Delta} \cdot \mathbf{M}
$$

where $\mathbf{M}$ is a diagonal matrix with the values of the mask on its main diagonal and $\boldsymbol{\Delta}$ is the DFT coefficients matrix introduced in (8). Also by $\operatorname{diag}(\cdot)$, we mean a diagonal matrix with diagonal elements as given inside the parenthesis.

This matrix multiplication for the signal and impulsive noise can be separately considered. First, the behavior of the signal through the iterations is examined. By using (14), (13) can be written as

$$
\mathbf{z}^{(k+1)}=(\mathbf{I}-\lambda \mathbf{G}) \mathbf{z}^{(k)}
$$

The spectrum of the signal, after multiplying by the mask, spreads over the frequency; hence, by the use of a lowpass filter, we moderately improve the distorted signal as the original signal is assumed to be lowpass. However, Amini in [30], [31] has shown that for matrices in the same form as $\mathbf{G}$, the iterative method converges even without the lowpass filtering. Consequently, the role of the filter can be viewed as an accelerating tool. Thus, in order to obtain a crude approximation of the convergence rate, we ignore the lowpass filter. Hence, (18) can be rewritten as

$$
\begin{aligned}
\mathbf{z}^{(k+1)} & =(\mathbf{I}-\lambda \mathbf{M}) \mathbf{z}^{(k)} \\
& =(\mathbf{I}-\lambda \mathbf{M})^{k+1} \mathbf{z}^{(0)}
\end{aligned}
$$

Since all the elements of $\mathbf{I}-\lambda \mathbf{M}$ are between zero and one, $\mathbf{z}^{(k)} \rightarrow 0$ as $k \rightarrow \infty$

Let $m_{i}$ be the $i^{\text {th }}$ element of the main diagonal of $\mathbf{M}$. After $l$ iterations, the $i^{\text {th }}$ element of the $(\mathbf{I}-\lambda \mathbf{M})^{l}$ is $(1-$ $\left.\lambda m_{i}\right)^{l}=e^{l \cdot \ln \left(1-\lambda m_{i}\right)}$; thus, the $i^{t h}$ element on the diagonal decays exponentially as $l$ increases with the time constant $\frac{1}{\tau_{i}}=\ln \left(\frac{1}{1-\lambda m_{i}}\right)$. To have a single time constant, we use the following average:

$$
\frac{1}{\tau_{a v}}=E\left\{\sum_{i} \frac{1}{\tau_{i}}\right\}
$$

where $\tau_{a v}$ is the average time constant and $E\{\cdot\}$ denotes the expected value operator.

To continue our analysis, we focus on the mask values. Using exponential soft-decision method, we have:

$$
m_{i}=e^{-\alpha|r[i]-\hat{s}[i]|}= \begin{cases}e^{-\alpha|s[i]+e[i]-\hat{s}[i]|} & r[i] \text { is noisy } \\ e^{-\alpha|s[i]-\hat{s}[i]|} & \text { otherwise }\end{cases}
$$

where $\hat{s}$ is the estimate of $s$ and $e$ represents the impulsive noise amplitude. In order to calculate $\tau_{a v}$, the Probability Distribution Function (PDF) of $s-\hat{s}$ and $s-\hat{s}+e$ should be calculated. We assume that the input signal has zero-mean Gaussian distribution. Since the estimation that we use are linear, $s[i]-\hat{s}[i]$ has also zero-mean Gaussian distribution with the variance of $\sigma_{\text {est }}^{2}$. With a similar assumption about the noise distribution (zero-mean Gaussian with variance $\sigma_{e}^{2}$ ) and independence of the noise and the signal, $s-\hat{s}+e$ also has zero-mean Gaussian distribution with variance $\sigma_{n}^{2}$. By the numerical calculation of $\tau_{a v}$ in the simulations, we have found out that the best linear fit with respect to the minimum mean squared error criterion is:

$$
\tau_{a v}=5.43+15 \alpha\left(p \sigma_{n}+(1-p) \sigma_{e s t}\right)
$$


where $p$ is the probability that a sample contains an impulsive noise. Now we can write the following approximation:

$$
\begin{gathered}
\mathbf{z}^{(l)}=\underbrace{(\mathbf{I}-\lambda \mathbf{M})^{l}}_{\approx e^{-l / \tau_{a v} \cdot \mathbf{I}}} \cdot \mathbf{z}^{(0)} \\
\Rightarrow\left\|\mathbf{z}^{(l)}\right\| \approx e^{-\frac{l}{\tau_{a v}}}\left\|\mathbf{z}^{(0)}\right\|
\end{gathered}
$$

In other words, excluding the impulsive noise, the improvement of the signal in each iteration can be approximated by $20 \tau_{a v}^{-1} \log (e) d B$.

Now we consider the effect of the iterations on the noisy part. We decompose the noise into lowpass and highpass parts as follows:

$$
\begin{aligned}
& \mathbf{e}_{L P}=\left(\boldsymbol{\Delta}^{-1} \cdot \operatorname{diag}(\underbrace{1, \ldots, 1}_{n-n_{z}}, \underbrace{0, \ldots, 0}_{n_{z}}) \cdot \boldsymbol{\Delta}\right) \cdot \mathbf{e} \\
& \mathbf{e}_{H P}=\left(\boldsymbol{\Delta}^{-1} \cdot \operatorname{diag}(\underbrace{0, \ldots, 0}_{n-n_{z}}, \underbrace{1, \ldots, 1}_{n_{z}}) \cdot \boldsymbol{\Delta}\right) \cdot \mathbf{e}
\end{aligned}
$$

It is clear that $\mathbf{e}=\mathbf{e}_{L P}+\mathbf{e}_{H P}$. Since the nonzero frequency components of $\mathbf{e}_{L P}$ coincide with the original low pass signal, the iterative method cannot distinguish these two signals. In other words, if the soft-decision method with large number of iterations is used, $\mathbf{e}_{L P}$ will be a dominant additive part. On the other, due to the use of the lowpass filter, $\mathbf{e}_{H P}$ vanishes. Hence, at the end of $\infty$ iterations, we will have an additive noise part with variance:

$$
\begin{aligned}
& E\left\{\left\|\mathbf{e}_{L P}\right\|^{2}\right\}=E\left\{\mathbf{e}_{L P}^{H} \cdot \mathbf{e}_{L P}\right\} \\
& =E\left\{\mathbf{e}^{H} \cdot \boldsymbol{\Delta}^{-1} \cdot \operatorname{diag}(\underbrace{1, \ldots, 1}_{n-n_{z}}, \underbrace{0, \ldots, 0}_{n_{z}}) \cdot \boldsymbol{\Delta} \cdot \mathbf{e}\right\} \\
& =\operatorname{Tr}\left\{E\left\{\mathbf{e} \cdot \mathbf{e}^{H}\right\} \cdot \boldsymbol{\Delta}^{-1} \cdot \operatorname{diag}(1, \ldots, 1,0, \ldots, 0) \cdot \boldsymbol{\Delta}\right\} \\
& =p \cdot \sigma_{e}^{2} \cdot \operatorname{Tr}\left\{\boldsymbol{\Delta}^{-1} \cdot \operatorname{diag}(1, \ldots, 1,0, \ldots, 0) \cdot \boldsymbol{\Delta}\right\} \\
& =\left(n-n_{z}\right) p \sigma_{e}^{2}
\end{aligned}
$$

Thus the impulsive noise power after $\infty$ iterations is expected to decrease by $-10 \log \left(1-\frac{n_{z}}{n}\right) d B$ (since the average noise power before the iteration is $n p \sigma_{e}^{2}$ ). It should be emphasized that if hard-decision is used (at higher RDE steps), the above equations are not valid and noise analysis should be performed by checking the error probability of the location detector. Another issue which is also important is that the above equations are comparing the noise before and at the end of $\infty$ iterations. In fact, the power of the noise after the first iteration is lower than the power of the noise after the last iteration; however, the quality of the signal is remarkably improved after the last iteration. Therefore, we expect to get a net gain in the signal to impulsive noise ratio after a finite number of iterations. Empirically, each iteration improves the signal component more than the degradation due to the impulsive noise reconstruction.

We finish this section by investigating the effect of the mask on the noisy part. Since the noise is impulsive, only the value of the mask at the locations of the impulses are important. For an impulse at the $i^{\text {th }}$ sample, the value of the mask is equal to $e^{-\alpha|s[i]+e[i]-\hat{s}[i]|}$. Thus the amplitude of this impulse after multiplication by the mask is

$$
\tilde{e}[i]=e[i] \cdot e^{-\alpha|s[i]+e[i]-\hat{s}[i]|}
$$

We have previously assumed that both noise and $s[i]-\hat{s}[i]$ are Gaussian distributed random variables with zero mean and variances $\sigma_{e}^{2}$ and $\sigma_{e s t}^{2}$, respectively. Thus:

$$
\begin{aligned}
E\left\{\tilde{e}^{2}[i]\right\}= & \int_{-\infty}^{\infty} \int_{-\infty}^{\infty} n^{2} \cdot e^{-2|x+n|} \frac{e^{-\frac{x^{2}}{2 \sigma_{e s t}^{2}}} \cdot e^{-\frac{n^{2}}{2 \sigma_{e}^{2}}}}{2 \pi \sigma_{e} \sigma_{e s t}} d x \cdot d n \\
< & \int_{-\infty}^{\infty} \int_{-\infty}^{\infty} n^{2} \cdot e^{2|x|-2|n|} \frac{e^{-\frac{x^{2}}{2 \sigma_{e s t}^{2}}} \cdot e^{-\frac{n^{2}}{2 \sigma_{e}^{2}}}}{2 \pi \sigma_{e} \sigma_{e s t}} d x \cdot d n \\
= & \int_{-\infty}^{\infty} n^{2} \cdot e^{-2|n|} \frac{e^{-\frac{n^{2}}{2 \sigma_{e}^{2}}}}{\sqrt{2 \pi} \sigma_{e}} \cdot d n \\
= & \int_{-\infty}^{\infty} e^{2|x|} \frac{e^{-\frac{x^{2}}{2 \sigma_{e s t}^{2}}}}{\sqrt{2 \pi} \sigma_{\text {est }}} \cdot d x \\
= & \sigma_{e}^{2}\left(e^{2 \sigma_{e}^{2}} Q\left(2 \sigma_{e}\right)\left(8 \sigma_{e}^{2}+2\right)-\frac{4 \sigma_{e}}{\sqrt{2 \pi}}\right) \\
\quad & \left(2 e^{2 \sigma_{e s t}^{2}} Q\left(-2 \sigma_{\text {est }}^{2}\right)\right) \\
\approx & \sigma_{e}^{2}\left(e^{2 \sigma_{e}^{2}} Q\left(2 \sigma_{e}\right)\left(8 \sigma_{e}^{2}+2\right)-\frac{4 \sigma_{e}}{\sqrt{2 \pi}}\right) e^{2 \sigma_{\text {est }}^{2}} \\
< & 0.41 \sigma_{e}^{2} \cdot e^{2 \sigma_{e s t}^{2}}
\end{aligned}
$$

where

$$
Q(x)=\int_{x}^{\infty} \frac{e^{-\frac{y^{2}}{2}}}{\sqrt{2 \pi}} \cdot d y
$$

Hence, when a good estimator is present $\left(\sigma_{e s t}<\frac{2}{3}\right)$, the variance of the masked noise falls below the variance of the noise before the mask:

$$
E\left\{\tilde{e}^{2}[i]\right\}<\sigma_{e}^{2}
$$

Furthermore, by lowpass filtering the masked output, this power is decreased again by discarding the high frequency components. To summarize, we have shown that the SNR of the reconstructed signal is at least:

$$
S N R_{\min }=\frac{N \sigma_{s}^{2}}{\left(n-n_{z}\right) p \sigma_{e}^{2}}
$$

where $\sigma_{s}$ is the standard deviation of the signal.

\section{Vi. An Alternative Method to Detect Impulsive NOISE LOCATIONS}

In [32], a decoding technique for DFT-based error control codes, using ELP is devised. In this section we combine the ELP decoding with the proposed iterative method. Let us assume that the original signal $(s[i])$ is an $n$-point discrete signal with $n_{z}$ contiguous zeros in the DFT domain. Thus, from the coding point of view, we are using an $\left(n, n-n_{z}\right)$-code which has the ability to correct up to $t=\frac{n_{z}}{2}$ random errors. We denote the impulsive noise and the corrupted signals by $e[i]$ and $r[i]$, respectively. The impulsive noise, $e[i]$, is assumed to be non-zero at most at $\frac{n_{z}}{2}$ samples. The corrupted signal and the impulsive noise are related to each other by:

$$
\begin{aligned}
r[i] & =s[i]+e[i] \\
R[k] & =S[k]+E[k]
\end{aligned}
$$


where the capital letters denote the DFT domain. Assume that

$$
S[k]=0 \quad \text { for } \quad k_{0} \leq k \leq k_{0}+2 t-1
$$

When $n_{e} \leq t$ errors are present in the received block, the error locator polynomial is defined as

$$
\begin{aligned}
H(\Omega) & =\prod_{k=1}^{n_{e}}\left(\Omega-\exp \left(j \frac{2 \pi}{n} i_{k}\right)\right) \\
& =\sum_{k=0}^{t} h_{k} \cdot \Omega^{k}
\end{aligned}
$$

where $\left\{i_{k}\right\}_{k=1}^{n_{e}}$ are the location of errors and $h_{k}=0$ for $n_{e}<$ $k \leq t$ and $h_{n_{e}}=1$. In fact the roots of this polynomial are exponentially related to the location of errors; i.e., $H\left(\Omega_{k}\right)=0$ for $\Omega_{k}=\exp \left(j \frac{2 \pi}{n} i_{k}\right)$ :

$$
H\left(\Omega_{k}\right)=h_{t} \Omega_{k}^{t}+h_{t-1} \Omega_{k}^{t-1}+\ldots+h_{0}=0
$$

Using the above equalities for $1 \leq k \leq n_{e}$, we can write:

$$
\begin{aligned}
0 & =\sum_{k=1}^{n_{e}} H\left(\Omega_{k}\right) \cdot e\left(i_{k}\right) \cdot \exp \left(-j \frac{2 \pi \cdot r}{n} i_{k}\right) \\
& =h_{t} E[r-t]+h_{t-1} E[r+1-t]+\ldots+h_{0} E[r]
\end{aligned}
$$

The good point is that $E[k]=R[k]$ for $k_{0} \leq k \leq k_{0}+$ $2 t-1$, and therefore the value of $E[k] \mathrm{s}$ are known in this range. If we set $k_{0}+t \leq r \leq k_{0}+2 t-1$ in (38), we obtain a system of $t$ equations with $t+1$ unknowns ( $h_{k}$ coefficients). These equations yield a unique solution for the polynomial with the additional condition that the last nonzero $h_{k}$ is equal to one. Instead of matrix inversion, a more efficient method is the Berlekamp-Massey algorithm for solving these equations. Finding the coefficients, we have to search for the roots. Since the roots of $H(\Omega)$ are of the form $\exp \left(j \frac{2 \pi}{n} i_{k}\right)$, the inverse DFT (IDFT) of the $\left\{h_{k}\right\}_{k=0}^{t}$ can be used. Before performing IDFT, we have to pad $n-1-t$ zeros at the end of the $\left\{h_{k}\right\}_{k=0}^{t}$ sequence to obtain an $n$-point signal. We refer to the new signal (after IDFT) as $\left\{H_{k}\right\}_{k=0}^{n-1}$. Each zero in $\left\{H_{k}\right\}$ represents an error in $r[k]$ at the same location.

As we are using the above method in the field of real numbers, exact zeros of $\left\{H_{k}\right\}$ s are rarely observed. Consequently, we threshold the $\left|H_{k}\right|$ s to find the zeros. Alternatively, $\left|H_{k}\right| \mathrm{s}$ can be used as a mask for soft-decision; in this case, thresholding is not needed.

\section{HARD-DECISION AND FILTERING}

The simplest method to eliminate the impulsive noise, is to hard-limit the noisy signal and then apply a lowpass filter to the output of the hard-limiter. This method does not use the redundancy in the signal efficiently; nevertheless, it can be viewed as a crude reconstruction algorithm. To have the best performance, we optimize the threshold for the hardlimiter. Let us assume that the amplitudes of the signal and the impulsive noise have the PDF's $N\left(0, \sigma_{s}^{2}\right)$ and $N\left(0, \sigma_{e}^{2}\right)$, respectively. Moreover, $p$ denotes the probability that a sample is noisy and $\eta$ represents the threshold for hard-limiting. The probability of error is

$$
P_{e}=(1-p) e^{\frac{-\eta^{2}}{2 \sigma_{s}^{2}}}+p\left(1-e^{\frac{-\eta^{2}}{2\left(\sigma_{s}^{2}+\sigma_{e}^{2}\right)}}\right)
$$

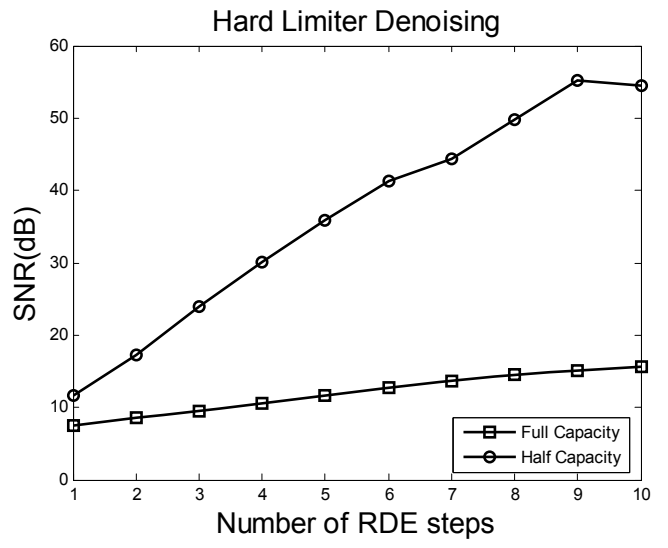

Fig. 4. Multiple Detection-Estimation steps using hard-decision and simple detector.

Then, the optimum threshold (to minimize $P_{e}$ ) can be derived by differentiating $P_{e}$ with respect to the threshold level:

$$
\frac{\partial}{\partial \eta} P_{e}=0
$$

hence,

$$
\eta_{o p t}=\sigma_{s} \sqrt{2(1+S N R) \cdot \ln \left(\frac{1-p}{p} \cdot \frac{1+S N R}{S N R}\right)}
$$

where $S N R=\sigma_{e}^{2} / \sigma_{s}^{2}$. In order to have an optimum threshold, $p$ should satisfy the following equation

$$
p \leq \frac{1+S N R}{1+2 S N R}
$$

\section{Simulation RESUltS}

To prove the effectiveness of the proposed method, various simulations are conducted. In all simulations, the signal is a filtered white Gaussian pseudo-random signal. We have also tested signals with uniform distribution which yielded similar results.

Fig. 4 show the simulation results when the process of hardlimiting and lowpass filtering is repeated. For the number of impulses, we considered both full capacity (half the number of DFT zeros of the signal) and half capacity (one fourth the number of DFT zeros of the signal). It is observed that in case of full capacity, the improvement after $10 \mathrm{RDE}$ steps is below $10 \mathrm{~dB}$.

As previously predicted, the soft thresholding method explained in section III, with repetition of detection and estimation processes, as shown in Fig. 5, yields much better results for full capacity (maximum number of impulses that can be corrected). For finding the mask function, both the simple soft method and CFAR are used. Fig. 5 suggests that by adding CFAR to the soft-decision, the recovery is enhanced. When we use CFAR, we require fewer number of iterations in the amplitude estimation steps of the impulsive noise; thus, CFAR adds little computational overhead on the overall complexity of the algorithm. 


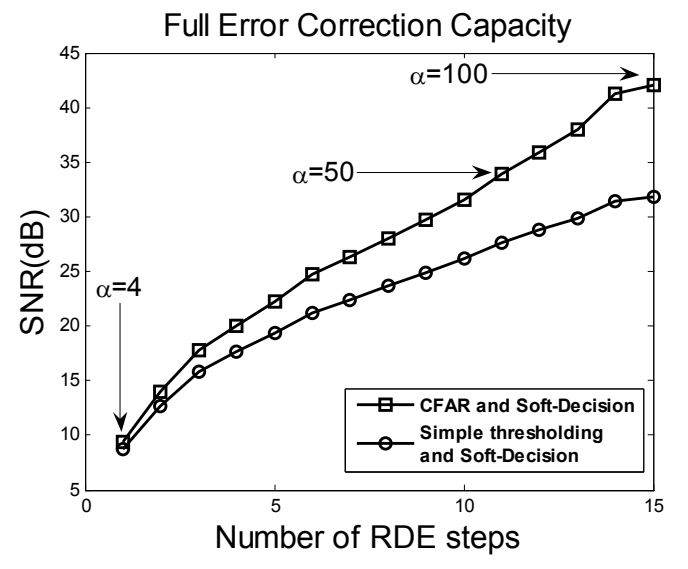

Fig. 5. Comparison of CFAR and simple soft-decision at full capacity.

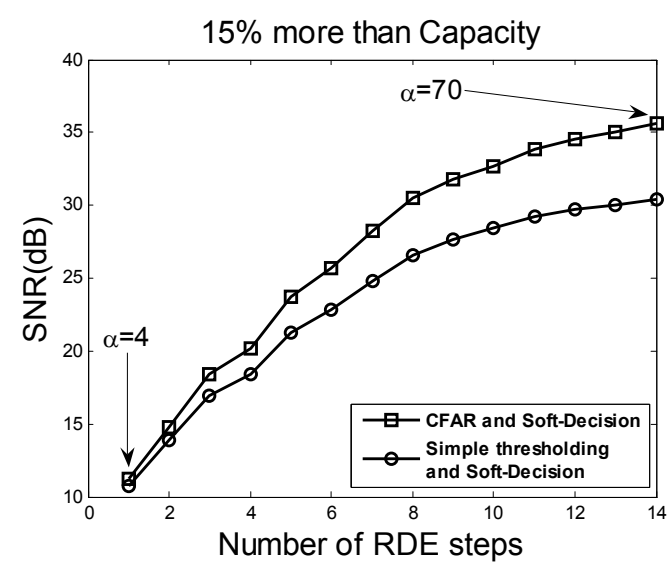

Fig. 6. SNR of the denoised signal when the error rate exceeds the reconstruction capacity by about $15 \%$.

The $\alpha$ parameter in the soft-decision method and the number of iterations in the estimator of the proposed method are varied from one RDE step to another as shown in Table I. These numbers are derived empirically and are not claimed to be optimal. As it is observed, the $\alpha$ parameter is non-decreasing, thus the decision rule is gradually changed from soft to a hard-decision as RDE steps proceed. Incidentally, the number of iterations in the estimator increases as the signal estimate becomes more reliable at higher steps. The CFAR parameters, $n_{c}$ and $k$ where chosen 20 and 15 . In the iterative method, parameter $\lambda$ is set to one.

If the number of impulses exceeds the reconstruction capacity, the SNR of the reconstructed signal is degraded gracefully since the iterative method leads to the pseudo-inverse solution [30], [31]. Fig. 6 depicts the SNR of the reconstructed signal when number of the errors introduced by the impulsive channel is $15 \%$ more than the theoretical reconstruction capacity. It can be seen that the SNR degrades gracefully compared to that of Fig. 5 but the algorithm does not diverge.

We have also simulated the ELP method in section VI to detect error locations and estimated the signal using 500

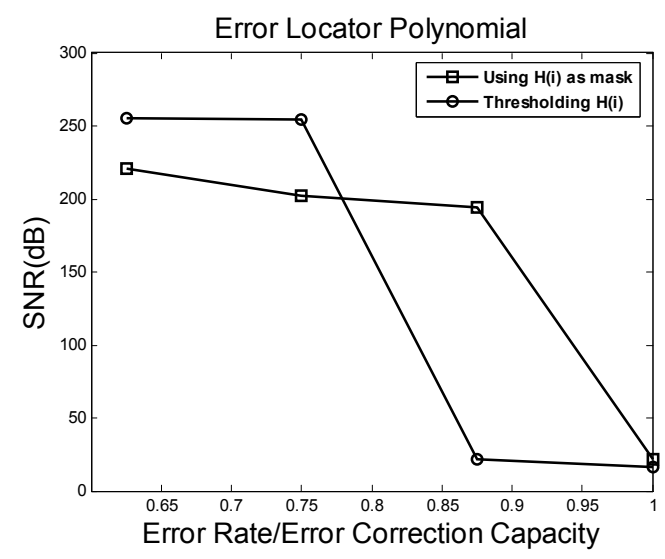

Fig. 7. SNR of the denoised signal using ELP to generate mask.

iterations. With the use of the Chebychev acceleration method [33], we can reduce this number to one third. For hard-decision ELP method, we have generated the mask by thresholding $\left|H_{k}\right|$ which has yielded a binary mask. The threshold is set to a small number to determine the locations of zeros. for $\left|H_{k}\right| \mathrm{s}$ smaller than the threshold, an error is assumed at that location. For soft-decision ELP method, we use $\left|H_{k}\right|$ as the mask. The performance of these methods are depicted in Fig. 7.

These methods do not perform as well as the previous method at the full capacity; however, it performs better for low error rates.

In Table II, we compare the relative complexity (computer time) and achievable SNR's for various methods at different impulsive noise rates. We have used thresholding in conjunction with lowpass filtering as the most simple reconstruction method (with complexity 1), and the complexity of other methods are compared to this simple method. As another simple method, median filtering is also used for comparison. This table shows clearly that at full capacity the soft-decision with the iterative technique gives the best performance in terms of SNR; the performance of ELP is less than half in SNR with 10 times more in complexity. However, at half the capacity, ELP performs very well.

We have initially assumed that each impulse corrupts only one sample of the signal. However, due to the characteristics of noise source, it is possible that consecutive impulses (burst error) occur. By increasing the number of consecutive impulses, the condition number of the matrix $G$ (introduced in section $\mathrm{V}$ ) also increases and consequently the convergence rate decreases. For matrices with poor condition number, additive white noise or finite numerical precision may lead to divergence. In our simulations, we can recover bursts of size 4 .

If prior to the transmission of the signal, characteristics of the noise source (channel) are known, interleaving in time or frequency (Sorted DFT [34]) can improve the robustness of the method in case of bursty impulses. The ELP method introduced in section VI could also be used to find the errors using Sorted DFT. 
TABLE I

SOFT-DECISION PARAMETER AND NUMBER OF ITER ATIONS

\begin{tabular}{|c|c|c|c|c|c|c|c|c|c|c|c|c|c|c|c|}
\hline RDE step & 1 & 2 & 3 & 4 & 5 & 6 & 7 & 8 & 9 & 10 & 11 & 12 & 13 & 14 & 15 \\
\hline$\alpha$ & 4 & 6 & 10 & 10 & 14 & 20 & 20 & 25 & 30 & 40 & 50 & 60 & 70 & 70 & 100 \\
\hline No. of Iterations & 50 & 50 & 50 & 50 & 100 & 100 & 100 & 100 & 100 & 100 & 200 & 200 & 200 & 200 & 200 \\
\hline
\end{tabular}

TABLE II

RELATIVE COMPLEXITY OF THE METHODS AND THE RESULTING SNR

\begin{tabular}{|c|c|c|c|c|}
\hline Method & $\begin{array}{c}\text { SNR }(\mathrm{dB}) \text { at } \\
\text { full capacity }\end{array}$ & $\begin{array}{c}\text { SNR(dB) at } \\
15 \% \text { overloaded }\end{array}$ & $\begin{array}{c}\text { SNR(dB) at } \\
70 \% \text { capacity }\end{array}$ & Complexity \\
\hline \hline Thresholding and LPF & 7 & 6.8 & 10 & 1 \\
\hline Median Filter & 18 & 13.5 & 20 & 1000 \\
\hline Non-Adaptive Hard-dec. & 13 & 9.5 & 19 & 500 \\
\hline CFAR Soft-dec & 42 & 35.1 & 50 & 5000 \\
\hline ELP & 20 & 2 & 250 & 50000 \\
\hline
\end{tabular}

\section{CONCLUSION}

In this paper, we proposed a new method for impulsive noise cancellation. In this method, with use of recursive detection/estimation steps, we approach the theoretical upper bound of reconstruction capacity. The SNR of the reconstructed signal can be improved by increasing the number of detection/estimation steps and increasing the number of iterations in each estimation block. Soft-decision and adaptive thresholding (CFAR) are used in the detection procedure which enhances the performance. Simulation results show that the SNR of the reconstructed signal degrades gradually if the number of impulses exceeds the theoretical reconstruction capacity. We have also used the ELP method in conjunction with the iterative method to estimate the corrupted samples of the signal. The SNR performance of the ELP method is higher than the RDE method for low error rates but much worse at full capacity, with a complexity that is higher by an order of magnitude.

The ideas used in this paper can also be used to other real field error correcting codes (DCT, wavelet and random codes), Sparse Component Analysis (SCA), and OFDM clipping noise removal.

\section{REFERENCES}

[1] M. Zimmermann and K. Dostert, "Analysis and modeling of impulsive noise in broad-band powerline communications," Electromagnetic Compatibility, IEEE Transactions on, vol. 44, no. 1, pp. 249-258, 2002.

[2] D. Levey and S. McLaughlin, "The statistical nature of impulse noise interarrival times in digital subscriber loop systems," Signal Processing, vol. 82, no. 3, pp. 329-351, 2002.

[3] M. Sanchez, L. de Haro, M. Ramon, A. Mansilla, C. Ortega, and D. Oliver, "Impulsive noise measurements and characterization in a UHF digitalTV channel," Electromagnetic Compatibility, IEEE Transactions on, vol. 41, no. 2, pp. 124-136, 1999.

[4] D. Middleton, "Non-gaussian noise models in signal processing for telecommunications: new methods an results for class A and class B noisemodels," Information Theory, IEEE Transactions on, vol. 45, no. 4, pp. 1129-1149, 1999.

[5] A. Spaulding and D. Middleton, "Optimum reception in an impulsive interference environment-Part I: Coherent detection," Communications, IEEE Transactions on [legacy, pre-1988], vol. 25, no. 9, pp. 910-923, 1977.

[6] C. Tepedelenlioglu and P. Gao, "On diversity reception over fading channels with impulsive noise,' Vehicular Technology, IEEE Transactions on, vol. 54, no. 6, pp. 2037-2047, 2005.
[7] T. Pander, "A suppression of an impulsive noise in ECG signal processing," IEEE Conf. of Engineering in Medicine and Biology Society (EMBC), vol. 1, pp. 596-599, 2004.

[8] R. C. Gonzalez and R. E. Woods, Digital Image Processing. PrenticeHall, Inc., 2002.

[9] R. Chan, C. Ho, and M. Nikolova, "Salt-and-pepper noise removal by median-type noise detectors and detail-preserving regularization," Image Processing, IEEE Transactions on, vol. 14, no. 10, pp. 1479-1485, 2005.

[10] D. Ze-Feng, Y. Zhou-Ping, and X. You-Lun, "High probability impulse noise-removing algorithm based on mathematical morphology," Signal Processing Letters, IEEE, vol. 14, no. 1, pp. 31-34, 2007.

[11] W. Luo, "An efficient detail-preserving approach for removing impulse noise in images," IEEE Signal Process. Lett. v13 i7, pp. 413-416.

[12] C.-B. Wu, B.-D. Liu, and J.-F. Yang, "A fuzzy-based impulse noise detection and cancellation for real-time processing in video receivers," Instrumentation and Measurement, IEEE Transactions on, vol. 52, no. 3 , pp. 780-784, 2003.

[13] I. Andreadis and G. Louverdis, "Real-time adaptive image impulse noise suppression," Instrumentation and Measurement, IEEE Transactions on, vol. 53, no. 3, pp. 798-806, 2004.

[14] S. Schulte, M. Nachtegael, V. De Witte, D. Van der Weken, and E. Kerre, "A fuzzy impulse noise detection and reduction method," Image Processing, IEEE Transactions on, vol. 15, no. 5, pp. 1153-1162, 2006

[15] E. Abreu, M. Lightstone, S. Mitra, and K. Arakawa, "A new efficient approach for the removal of impulse noise fromhighly corrupted images,' Image Processing, IEEE Transactions on, vol. 5, no. 6, pp. 1012-1025, 1996.

[16] H. Eng and K. Ma, "Noise adaptive soft-switching median filter," Image Processing, IEEE Transactions on, vol. 10, no. 2, pp. 242-251, 2001.

[17] P. Ferreira and J. Vieira, "Stable DFT codes and frames," Signal Processing Letters, IEEE, vol. 10, no. 2, pp. 50-53, 2003.

[18] F. Marvasti, Nonuniform Sampling: Theory and Practice. Kluwer Academic/Plenum Publishers, 2001.

[19] P. Azmi and F. Marvasti, "A pseudo-inverse based iterative decoding method for DFT codes in erasure channels," IEICE TRANSACTIONS on Communications, vol. 87, no. 10, pp. 3092-3095, 2004.

[20] R. E. Blahut, Algebraic Codes for Data Transmission. Cambridge University Press, 2003.

[21] F. Marvasti and M. Nafie, "Using FFT for error correction decoders," DSP Applications in Communication Systems, IEE Colloquium on, p. 9, 1993.

[22] J. Wu and J. Shin, "Discrete cosine transform in error control coding," Communications, IEEE Transactions on, vol. 43, no. 5, pp. 1857-1861, 1995.

[23] P. Ferreira, "Stability issues in error control coding in the complex field, interpolation, and frame bounds," Signal Processing Letters, IEEE, vol. 7, no. 3, pp. 57-59, 2000.

[24] M. Skolnik, Introduction to Radar Systems. McGraw-Hill, 2000.

[25] N. Nedev, S. McLaughlin, and D. Laurenson, "Estimating errors in transmission systems due to impulse noise," IEE ProceedingsCommunications, vol. 153, p. 651, 2006. 
[26] D. Middleton, "Statistical-physical models of electromagnetic interference," Electromagnetic Compatibility, IEEE Transactions on, pp. 106127, 1977.

[27] M. Alamdari and M. Modarres-Hashemi, "An improved CFAR detector using wavelet shrinkage in multiple target environments," in 9th International Symposium in Signal Processing and its Applications, Sharjah,UAE, Feb 12-15 2007.

[28] S. Zahedpour, M. Ferdosizadeh, F. Marvasti, G Mohimani, and M. Babaie-Zadeh, "A novel impulsive noise cancellation based on successive approximations," in Proceedings of SampTa2007, Thessaloniki, Greece, June 2007.

[29] A. Amini, M. Babaie-Zadeh, and C. Jutten, "A fast method for sparse component analysis based on iterative detection-estimation," AIP Conference Proceedings, vol. 872, p. 123, 2006.

[30] A. Amini and F. Marvasti, "Reconstruction of multiband signals from non-invertible uniform and periodic nonuniform samples using an iterative method," in Proceedings of SampTa2007, Thessaloniki, Greece, June 2007.

[31] - - "Convergence analysis of an iterative method for the reconstruction of multi-band signals from their uniform and periodic nonuniform samples," to appear in Sampling Theory in Signal and Image Processing (special issue in nonuniform sampling), vol. 7, no. 2, May 2008.

[32] P. Azmi and F. Marvasti, "A novel decoding procedure for real field error control codes in the presentation of quantization noise," Proc. IEEE Conference on Acoustic Speech, and Signal Processing ICASSP2003, vol. IV, pp. 265-268, 2003

[33] K. Grochenig, "Acceleration of the frame algorithm," Signal Processing, IEEE Transactions on [see also Acoustics, Speech, and Signal Processing, IEEE Transactions on], vol. 41, no. 12, pp. 3331-3340, 1993.

[34] F. Marvasti, "Error concealment or correction of speech, image and video signals," United States Patent 6,601,206, July 2003. 